EUROPEAN ORGANIZATION FOR NUCLEAR RESEARCH

CERN-EP/2001-032

4 April 2001

\title{
A MEASUREMENT OF LORENTZ ANGLE AND SPATIAL RESOLUTION OF RADIATION HARD SILICON PIXEL SENSORS
}

\author{
I. Gorelov, G. Gorfine, M. Hoeferkamp, S. C. Seidel \\ New Mexico Center for Particle Physics, University of New Mexico, Albuquerque, \\ NM-87131, USA
}

A. Ciocio, K. Einsweiler, M. Gilchriese , A. Joshi, S. Kleinfelder, R. Marchesini, O. Milgrome, N. Palaio, F. Pengg, J. Richardson, G. Zizka

E. O. Lawrence Berkeley National Laboratory and University of California, Berkeley, CA-94720, USA

M. Ackers, P. Fischer, M. Keil, S. Meuser, T. Stockmanns, J. Treis, N. Wermes Physikalisches Institut, Universität Bonn, D-53115 Bonn, Germany

C. Gößling, F. Hügging, J. Wüstenfeld, R. Wunstorf

Institut für Physik, Universität Dortmund, D-44221 Dortmund, Germany

D. Barberis, R. Beccherle, M. Cervetto, G. Darbo, G. Gagliardi, C. Gemme, P. Morettini, P. Netchaeva, B. Osculati, F. Parodi, L. Rossi

Dipartimento di Fisica, Universita' di Genova e I.N.F.N. Sezione di Genova, I-16146

Genova, Italy

K. Dao, D. Fasching

Department of Physics, University of Wisconsin, WI 53706 Madison, USA

L. Blanquart, P. Breugnon, D. Calvet, J-C. Clemens, P. Delpierre, G. Hallewell, D. Laugier, T. Mouthuy, A. Rozanov, C. Trouilleau, I. Valin

Centre de Physique des Particules de Marseille, IN2P3-CNRS, F-13288 Marseille, France

M. Aleppo, A. Andreazza, M. Caccia, T. Lari, C. Meroni, F. Ragusa, C. Troncon*), G. Vegni

Dipartimento di Fisica, Universita' di Milano e I.N.F.N. Sezione di Milano, I-20133

Milano, Italy

T. Rohe ${ }^{a)}$

Max-Planck-Institut für Physik, D-80805 München, Germany

G. R. Boyd, H. Severini, P. L. Skubic, J. Snow

Department of Physics and Astronomy, University of Oklahoma, Norman, OK-73019

USA

P. Š́cho, L. Tomasek, V. Vrba

Academy of Sciences of the Czech Republic, Institute of Physics, CZ-18040 Prague, Czech Republic 


\begin{abstract}
Silicon pixel sensors developed by the ATLAS collaboration to meet LHC requirements and to withstand hadronic irradiation to fluences of up to $10^{15} \mathrm{n}_{\mathrm{eq}} / \mathrm{cm}^{2}$ have been evaluated using a test beam facility at CERN providing a magnetic field. The Lorentz angle was measured and found to alter from $9.0^{\circ}$ before irradiation, when the detectors operated at $150 \mathrm{~V}$ bias at $B=1.48 \mathrm{~T}$, to $3.1^{\circ}$ after irradiation and operating at $600 \mathrm{~V}$ bias at $1.01 \mathrm{~T}$. In addition to the effect due to magnetic field variation, this change is explained by the variation of the electric field inside the detectors arising from the different bias conditions.

The depletion depths of irradiated sensors at various bias voltages were also measured. At $600 \mathrm{~V}$ bias $280 \mu \mathrm{m}$ thick sensors depleted to $\approx 200 \mu \mathrm{m}$ after irradiation at the design fluence of $1 \times 10^{15} 1 \mathrm{MeV} \mathrm{n}$ eq $/ \mathrm{cm}^{-2}$ and were almost fully depleted at a fluence of $0.5 \times 10^{15} 1 \mathrm{MeV} \mathrm{n}$ eq $/ \mathrm{cm}^{-2}$.

The spatial resolution was measured for angles of incidence between $0^{\circ}$ and $30^{\circ}$. The optimal value was found to be better than $5.3 \mu \mathrm{m}$ before irradiation and $7.4 \mu \mathrm{m}$ after irradiation.
\end{abstract}

Keywords: Lorentz Angle, Spatial Resolution, Silicon Pixel Detectors , Radiation Hardness , Depletion Depth , LHC

PACS 29.40.G , 29.40.W , 85.30, 61.80.H

Submitted to Nuclear Instruments and Methods in Physics Research

*) Corresponding author. Address: Dipartimento di Fisica, Universita' di Milano e INFN, via Celoria 16, I-20133 Milano, Italy. E-mail:clara.troncon@mi.infn.it

Telephone: +3902 58357388, fax: +3902 58357624

a) Now at PSI, CH-5232 Villigen, Switzerland

b) Now at University of Glasgow, Glasgow G12 8QQ, UK 
At the LHC silicon microstrip and pixel detectors will be exposed to unprecedented levels of ionizing and hadronic radiation. As a consequence of displacement damage arising from hadronic radiation, the silicon detectors will change their characteristics during their lifetime, resulting in the requirement that their operating conditions (bias voltage, temperature etc.) be modified. These factors influence the motion of charge carriers and affect the charge collection performance. Potential radiation-induced performance degradation is a serious issue which has thus been studied in detail.

Spatial resolution and hit efficiency may be affected by variations in the amount of collected charge and in its sharing amongst neighbouring pixels. Optimal resolution is achieved when the charge generated by particles that traverse the sensor is spread over two pixels and an interpolation algorithm is used for the determination of the crossing point. Charge sharing is determined by the depth of the depleted region, the Lorentz angle, the angle of the sensor with respect to the incident particles and nonuniformity in charge collection.

In this paper measurements of the Lorentz angle and spatial resolution (as a function of the incident angle) of prototype ATLAS pixel sensors, before and after irradiation to fluences of up to $10^{15} \mathrm{n}_{\text {eq }} / \mathrm{cm}^{2}{ }^{1)}$, are presented.

A model [1] for charge drift in silicon has been developed and is used to predict the behaviour of silicon devices for comparison with the data. An essential parameter in this model is the value of depletion depth for irradiated devices and a new method to measure this quantity has been developed.

\section{ATLAS PIXEL DETECTOR}

A full description of the ATLAS pixel detector can be found in [2], so only a brief description is given here.

\section{$2.1 \quad$ Pixel sensors}

The ATLAS pixel sensors consist of $n^{+}$implants on a high resistivity $n$-bulk substrate [3]. This choice was taken because it allows for operation in partially depleted mode after type inversion of the silicon material and subsequent increases in the full depletion voltage, arising from radiation damage. Before irradiation the $p n$-junction is located on the sensor $p^{+}$-backside. After type inversion it moves to the segmented $n^{+}$pixel side of the sensor and the depleted region grows from the $n^{+}$pixel implants to the opposite side of the sensor, thus allowing collection of charge carriers on the pixels, even when the sensor is not fully depleted.

Two techniques of $n^{+}$implant isolation were incorporated in the prototype designs: the $p$-stop [4], where a high dose p-implant surrounds the $n$-cell and the $p$-spray [5] technique, where a uniform medium dose $p$-implant covers the whole $n$-side and is overcompensated by the high dose of the pixel implants themselves.

Two configurations of $p$-spray have been studied, in the first of these the dose is uniform over the whole sensor area. In the other configuration (known as moderated p-spray) the $p$-doping concentration is reduced in the regions surrounding $n^{+}$implants. In this way the electric field is reduced at the interface from $p$-type to $n^{+}$-type thus increasing the breakdown voltage.

1) In this paper the fluences are converted in $n_{e q} / \mathrm{cm}^{2}$ where $n_{e q}$ is the number of particles with the non-ionizing energy loss of a $1 \mathrm{MeV}$ neutron. 
The $p$-spray technique allows the introduction of a punch-through bias grid. This is useful for sensor testing prior to connection to front-end electronics through bump-bonding. This is implemented every 2 nd column, providing an equal potential to every pixel cell during testing; it is inactive in normal operation, but keeps a pixel cell close to ground potential in the case that a bump may be missing. The backside of the sensor has a multi guard ring structure which serves to grade the potential down towards the edges. Using $n^{+}$pixel implants on $n$-type substrate allows to have a continuous $n^{+}$-implant outside the active area without a guard ring structure on the $n$-side. The edges of the sensor are thus held at ground, which prevents arcing between the detector and the front-end electronics.

Wafers containing two or three sensor tiles of the size to be used for ATLAS pixel modules $\left(16.4 \times 60.8 \mathrm{~mm}^{2}\right.$ and to be bump bonded to an array of $2 \times 8$ electronic chips $)$ and several sensors sized to accept single electronics chip $\left(7.4 \times 8.2 \mathrm{~mm}^{2}\right)$ were fabricated in $200 \div 300 \mu \mathrm{m}$ thicknesses by C.i.S. (Germany) ${ }^{2)}$ and Seiko (Japan) ${ }^{3)}$. The result$\mathrm{s}$ reported in this paper refer to these smaller sized sensors. They incorporated various designs (Fig. 1), developed to study and thus optimise the performance in terms of interpixel capacitance, cross-talk, noise, efficiency and intrinsic resolution. Some of these designs match the tile designs and are referred to as ST1 (Single Tile 1), ST2 (Single Tile 2), SSG (Single Small Gap) and SMD (Small Dot).

$P$-stop isolation was adopted in ST1, while p-spray isolation was used in all of the other

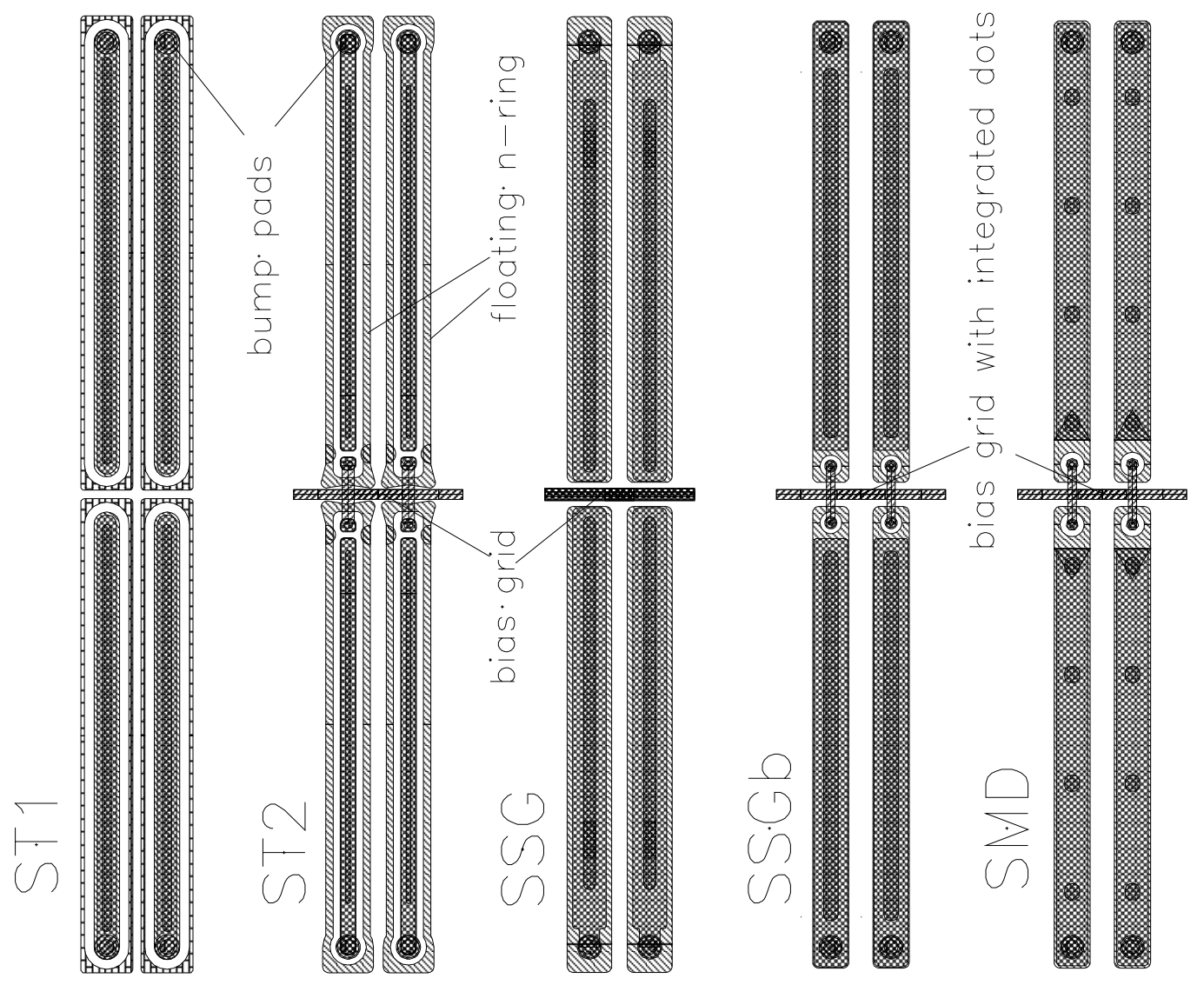

Figure 1: Tested design options of the ATLAS pixel sensors.

2) C.i.S. Centre for intelligent Sensors, Erfurt (Germany)

3) Seiko Instruments Inc., Chiba (Japan) 
designs. In the ST1 design the $p^{+}$-type isolation rings surround the $n^{+}$-pixel implants. In the ST2 design there are $n^{+}$-type ring-like structures around each pixel to reduce the inter-pixel capacitance. The SSG design uses $p$-spray isolation but does not include any intermediate $n^{+}$structures. Neighbouring pixels merely have small gaps between them. Since the ST2 design had a charge collection problem related to the presence of the floating $n^{+}$ring structure surrounding the pixel, an SSG-like design was adoped as the baseline, with some modifications in the region of bias grid (SSGb design). In a subsequent sensor production this SSGb design has been labelled SMD. In the SMD-design each pixel consists of one rectangular $n^{+}$-implantation of dimensions $30 \mu \mathrm{m} \times 382.5 \mu \mathrm{m}$. The gap between two adjacent pixel is $20 \mu \mathrm{m}$ in the short pitch direction, $15 \mu \mathrm{m}$ in the long pitch direction at the pixel side with the bump-pad and $20 \mu \mathrm{m}$ at the other pixel side where the bias-grid is located. The bias-grid connection to each pixel is provided using a round $n^{+}$ implant integrated in the $n^{+}$-pixel implant with $10 \mu \mathrm{m}$ diameter and $5 \mu \mathrm{m}$ gap (to limit the coupling from the bias dot to that particular pixel). The bias-dots of all pixels within an adjacent pair of columns are connected via a metal line to an outer $n^{+}$-implantation which surrounds the border of the active sensor area.

Sensors fabricated on both standard detector-grade and oxygenated [6] silicon substrates have been evaluated.

The effective dimensions of the pixel cell are $50 \mu \mathrm{m} \times 400 \mu \mathrm{m}$. They are connected via bump bonds to the readout electronics cells which are arranged in a matching matrix.

\section{$2.2 \quad$ Front-end electronics}

All of the pixel devices documented here were assembled using readout chips produced during the demonstrator phase of the ATLAS Pixel front-end electronics development programme. The aim of the demonstrator programme was to produce realistic readout-electronics which addressed all of the requirements and specifications of the ATLAS Pixel tracker. The initial phase of the programme resulted in the development of two separate front-end chips which although conceived with radiation hard processes in mind, were realised at non-radiation hard foundries. The first of the front-end chips, FEA [7], was fabricated using the BiCMOS process of AMS. The front-end of FE-A used bipolar transistors although a $100 \%$ CMOS version (called FE-C) was later produced. The design of the other chip, FE-B [8], was manufactured by HP. In each FE-chip, 2880 channels are arranged into 18 column by 160 rows. The chips have integrated DACs providing the necessary biases for the analogue front-end circuitry. Each channel is equipped with its own 3-bit DAC for channel-to- channel threshold adjustments, thus a means of overall dispersion reduction is provided. All of the ATLAS prototype FE chips have 7-bit charge measurement capability using time-over-threshold (TOT), taking advantage of the available deadtime per pixel ( $2 \mu$ s excepting the innermost ATLAS pixel layer known as B-layer). Also featured is a global hit-OR (hitbus) which provides for a means of self-triggering operation. A 2880-bit pixel register plus one corresponding latch per channel enable individual pixels to be masked-off for (independently) calibration-strobing and readout. The charge-sensitive preamplifiers feature a DC feedback scheme with a tuneable current providing control over the shaping-time for a given input charge. A discrimination stage sits behind the preamplifier in each channel which is sensitive to the leading edges (LE) and trailing edges (TE) of pulses.

The readout-architecture implementations are markedly different for the two designs. In FE-A/C each column-pair is served by an $80-$ bit $40 \mathrm{MHz}$ shift register to clock LE and 
TE hit-information towards the end-of-column (EOC) circuitry. There the timestamps of the hits are determined from the row-number information along with the time-of-arrival at the EOC. Since the length of the shift registers is 80-bits only one LE or TE from a 4-pixel cell may be introduced for a given BCO. If extra hits are registered in a particular crossing then their information is held until the next available crossing and a 2-bit latefield is used to indicate the required correction to the hit age at the EOC. In FE-B the global time information is distributed throughout the array to every pixel as 7-bit Graycode. When a hit is tagged in a pixel cell the timestamps for the LE and TE are stored locally. Meanwhile a continuous vertical-sparse-scan operates along the column pairs seeking tagged hits. As soon as such a hit is seen, the geographical and timing information is sent directly to the 20 buffer-sets which are resident at the end of each column-pair. Upon receipt of a level-1 trigger, a two-dimensional horizontal-sparse-scan looks through the EOC buffers for hits which match in time for the given latency (which is coded in a register on the chip). Matching hits are then stacked up in a readout-FIFO for subsequent serial transmission. The TOT is calculated for each hit as the TE-LE time-difference prior to this, resulting in a 7-bit charge field in each 26-bit hit-word.

During the operation in the test beam the thresholds of the individual channels were adjusted achieving a threshold dispersion of $120 e^{-}$rms. Typical thresholds were around 3000 electrons, with a noise of $110 e^{-}$for ST1 and ST2, $170 e^{-}$for SSG. The TOT was calibrated by injecting a known charge into every channel, with an accuracy of the order of $10 \%$.

\subsection{Hybridization}

The pixel sensor and FE electronics are electrically connected via bump bonds. Bump bonding at $50 \mu \mathrm{m}$ pitch was considered a serious challenge until recently. Hovewer several firms were able to provide high density bump bonding using both Indium and $\mathrm{PbSn}$ solder techniques with a connection failure rate lower than $10^{-4}$. For the Indium process, the bumps are grown both on the sensor and on the electronics chip. During flip-chipping the two surfaces are pressed together forming a cold weld. For solder, the bumps are grown only on electronics surface and the flip-chip step is performed at 250 ${ }^{\circ} \mathrm{C}$ in order to reflow the solder. Assemblies using both techniques have been evaluated in the test beam.

\subsection{Irradiated sensors}

In order to characterize irradiated sensors with pixel readout electronics, indium bumps were deposited on some single chip sensors which were then exposed to fluences comparable to those expected at the LHC. The irradiations were performed using the 300 $\mathrm{MeV} / c$ pion beam at PSI and the $55 \mathrm{MeV} / c$ proton beam at LBNL. After irradiating these devices, they were flip chipped to the front-end electronics. Since indium bumps do not need to be heated for flip chipping, this process was used to limit reverse annealing. Sensors irradiated with fluences of $0.5 \times 10^{15}$ and $1 \times 10^{15} \mathrm{n}_{\mathrm{eq}} / \mathrm{cm}^{2}$ were tested in the beam. They were cooled to $-9{ }^{0} \mathrm{C}$ during data taking.

Sensors with $p$-stop isolation (ST1) produced high noise even at low bias voltages. Irradiated sensors with $p$-spray separation, on the other hand, performed well at voltages of up to $600 \mathrm{~V}$. 
Test beam experiments were performed in 1998-2000 at the CERN SPS accelerator with a pion beam of $180 \mathrm{GeV} / \mathrm{c}$ momentum. A beam telescope consisting of 4 pairs of silicon microstrip detectors (each pair having two planes of detectors with orthogonal strips) was used to measure the transverse position of the incident beam particles. The position resolution of tracks projected onto the test devices varied from $3 \mu \mathrm{m}$ to $6 \mu \mathrm{m}$ depending on the position of the detector under test with respect to the telescope (see section 6). For the Lorentz angle measurements, the pixel assemblies and the silicon strip telescope were positioned inside a superconducting magnet providing a field of magnitude up to $1.67 \mathrm{~T}$. Data have been taken at various values of magnetic field. The pixels were oriented in such a way that the charge carrier motion in the $50 \mu \mathrm{m}$ pitch direction was influenced by the magnetic field.

Relevant parameters of some of the sensors tested in the beam may be found in Table 3. When not otherwise stated, they were fabricated in standard detector-grade silicon substrates.

\subsection{Data Analysis}

Events were filtered with the requirement of one and only one track reconstructed by the silicon microstrip telescope in each event. Tracks were required to extrapolate into a fiducial region inside the pixel sensor. This was defined as the region within $40 \mu \mathrm{m}$ from the edge of the pixel sensor for perpendicular tracks or at least $40+\mathrm{t} \times \operatorname{tg} \alpha \mu \mathrm{m}$ for inclined tracks, where $\mathrm{t}$ is the sensor thickness and $\alpha$ the incidence angle (such that $\alpha=0^{\circ}$ is normal incidence).

In addition, only events with track reconstruction $\chi^{2}$-probability value of more than 0.02 were kept. Pixel clusters were constructed in the following way:

- all pixel hits were considered independent of track extrapolations;

- all adjacent hits were clustered together.

For irradiated sensors at large incidence angles, the clustering algorithm allowed for the presence of not-hit pixels within a cluster.

The cluster position was then evaluated as described in section 6 .

To associate a cluster to a beam particle track the residual between track extrapolation and mean cluster position was required to be less than $100 \mu \mathrm{m}$ in the short pixel dimension and $800 \mu \mathrm{m}$ in the long pixel dimension. Clusters and events that passed all the above requirements were used in the measurements reported in this paper.

\section{DEPLETION DEPTH OF IRRADIATED SENSORS}

To perform measurements of the depletion-zone depth, data were taken with particle beam incident on the sensor at an angle of $20^{\circ}$ or $30^{\circ}$ with respect to the normal to the pixel plane (Fig. 2). A particle crossing the detector produces a cluster of hits consisting of the pixel cells that collect a significant fraction of the charge released in the subtended segment of the track. When the sensor is not fully depleted, the cluster width may be less than that expected by purely geometric considerations. To measure the depth of the depleted region the maximum depth of track segment was used (Fig. 2); it was defined as the distance of the center of the track-segment subtended by a given pixel from the pixel itself. Since the entrance points of the tracks are uniformly distributed, all of the depths vary continously and the maximum observed depth $\mathrm{D}$ is a measurement of the depletion depth within the detector. The distribution of the depths is shown in Fig. 3 for 


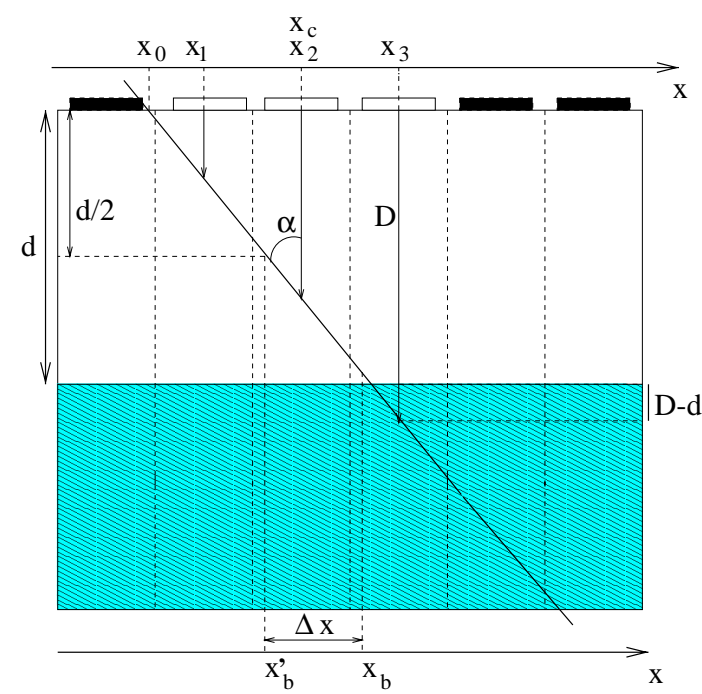

Figure 2: Schematic view of an irradiated sensor crossed by a track. The hatched zone corresponds to the not-depleted zone.

a sensor irradiated with a fluence of $0.5 \times 10^{15} \mathrm{n}_{\mathrm{eq}} / \mathrm{cm}^{2}$ for four different bias voltages. The observed distribution is a convolution of a uniform distribution between 0 and $\mathrm{D}$ and a resolution function due to extrapolation error of the beam telescope etc.

A fit to the data yields $\mathrm{D}$, defined as the point of inflection of the fitting function. Two main corrections are needed to correctly estimate the depletion depth. First the measurement of the position of the impact point $x_{0}$ of the beam particle into the pixel plane must be corrected for the relative alignments of the telescope planes and the pixel plane. The pixel sensors are aligned with respect to the silicon microstrip telescope by minimizing the residuals $x_{b}-x_{c}$, where $x_{b}$ is the position of the beam particle track extrapolated to the plane at the center of the sensor. $x_{c}$ is the cluster position, defined as the charge-weighted average of the positions of the hit pixel cells. The definitions of the two positions are inconsistent since a proper alignment would require extrapolation of the beam particle track to a plane at the center of the depleted region $\left(x_{b}^{\prime}\right)$ of the sensor whose depth is unknown: this causes a systematic offset $\Delta x$ to the alignment that must be accounted for. The residual misalignment $\Delta x$ between telescope and pixel coordinates is determined using the charge collected on the first pixel in the cluster. With reference to Fig. 4, pixel $n$ is the first hit pixel only when the impact position of the track is between the points $x_{A}$ and $x_{C}$. Point $x_{A}$ is defined by requiring that the charge collected by pixel $n-1$ is equal to the threshold. Similarly, point $x_{C}$ is defined by requiring that the charge collected by pixel $n$ is also equal to the threshold; in both cases the segment length $x_{A}-x_{B}=x_{C}-x_{D}=l$ is related to the electronics threshold. The charge collected on pixel $n$ as a function of the position of the impact point, $x_{E}$, of the beam particle is constant when $x_{E}$ lies between $x_{A}$ and $x_{B}$, but linearly decreases when $x_{E}$ lies between $x_{B}$ and $x_{C}$, as sketched in the upper part of Fig. 4. The position of the knee is the true position of the edge of the pixel and enables the systematic alignment correction $\Delta x$ to be determined. In Fig. 5b the ToT of the first pixel as a function of the distance of the impact point $x_{0}$ from the center of pixel $n$ is plotted for an unirradiated ST2 sensor.

The second correction deals with the effect of the threshold on the definition of the maximum observed depth, D. Referring again to Fig. 2 it can be seen that the maximum 

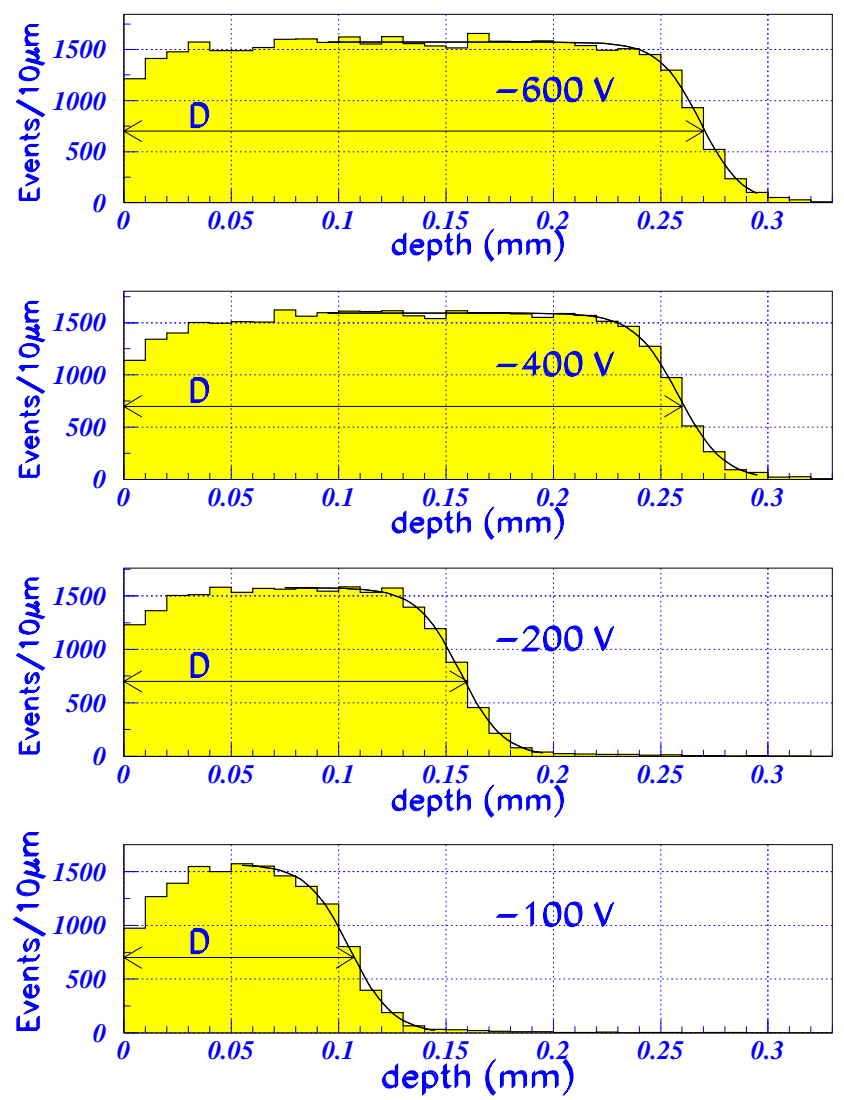

Figure 3: Distribution of the track-segment depth for sensors irradiated to a fluence of $0.5 \times 10^{15} \mathrm{n}_{\mathrm{eq}} / \mathrm{cm}^{2}$ for four different bias voltages.

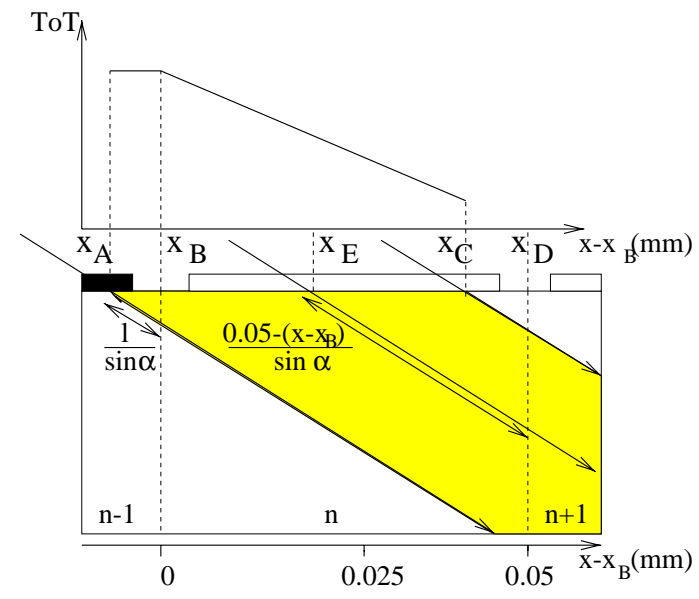

Figure 4: Position of the entry point of tracks that hit pixel $n$ but not pixel $n-1$. The expected shape of the Time over Threshold of the first hit pixel as a function of the incident track position is sketched above.

measured value of $\mathrm{D}$ occurs when the charge collected by the last pixel is just above the threshold. In this case the correction to the depth $\mathrm{D}$ is $\mathrm{D}-\mathrm{d}=(p / 2-l) / \operatorname{tg} \alpha, p$ being the pixel size $(50 \mu \mathrm{m})$ and $l$, defined above, the projected length of the track-segment 
corresponding to the threshold. To measure $l$, the distribution of the hits of pixel $n$ as a function of the distance of the impact point of the beam particle $x_{0}$ from its center was studied (Fig. 5a). The mean value of the distribution is not zero since pixel $n$ was hit also when $x_{0}$ was on pixel $n-1$. The measured mean value is the value of $l$.

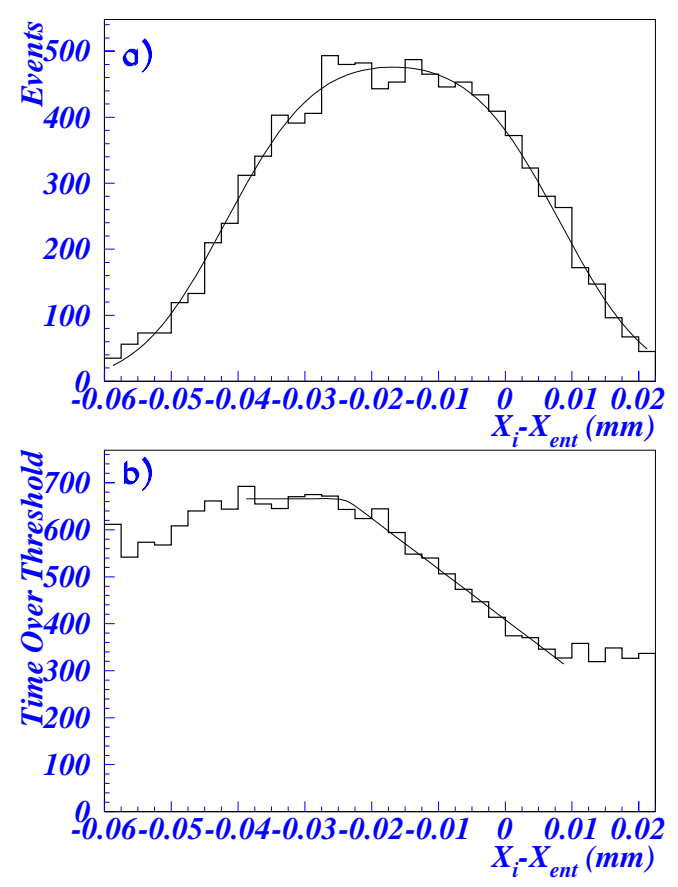

Figure 5: a): Distribution of track positions relative to the center of the first hit pixel. b): ToT of the first hit pixel as a function of track position relative to pixel center. The fitting functions are superimposed.

The consistency of the method was checked with fully depleted unirradiated sensors, for which the measured depletion depth should be equal to the nominal thickness. The measured depletion depth values were: $288 \pm 6 \mu \mathrm{m}$ for an unirradiated $280 \mu \mathrm{m}$ thick sensor and $190 \pm 7 \mu \mathrm{m}$ for an unirradiated sensor which was nominally $200 \mu \mathrm{m}$ thick. The errors are dominated by the uncertainty on the threshold corrections.

Table 1 summarizes the results of the measurements made on ST2 sensors irradiated with two different fluences. This table also shows results for unirradiated sensors of the ST2 and SSGb types. All ST2 sensors were $280 \mu \mathrm{m}$ thick while the SSGb sensor was $200 \mu \mathrm{m}$ thick. They were fabricated on standard silicon. These results show that after a fluence of $1 \times 10^{15} \mathrm{n}_{\text {eq }} / \mathrm{cm}^{2}$ an ST2 sensor had a depletion depth ${ }^{4)}$ of $\approx 200 \mu \mathrm{m}$ at $600 \mathrm{~V}$, while after a fluence of $0.5 \times 10^{15} \mathrm{n}_{\text {eq }} / \mathrm{cm}^{2}$ the same design was almost fully depleted at $600 \mathrm{~V}$. Table 1 also shows the result of a bias voltage scan performed on one of the tested sensors.

\section{DETERMINATION OF LORENTZ ANGLE}

In the presence of an electric field, $E$, and a magnetic field, $B$, the charge carriers liberated by a passing particle within silicon drift along a direction at an angle $\Theta_{L}$

4) The two values reported in Table 1 refer to the same sensor, evaluated at one year distance. The precision is not sufficient to conclude that the depletion depth had actually increased. There may have been an effect however due to annealing. 
( Lorentz angle) with respect to the electric field direction (due to the $\vec{E} \times \vec{B}$ effect ). They usually spread over several pixels, depending on the angle of incident particle. This spread is a minimum for an angle equal to the Lorentz angle as shown schematically in Fig. $6^{5)}$. Knowledge of this angle is needed to optimize the spatial resolution by tuning

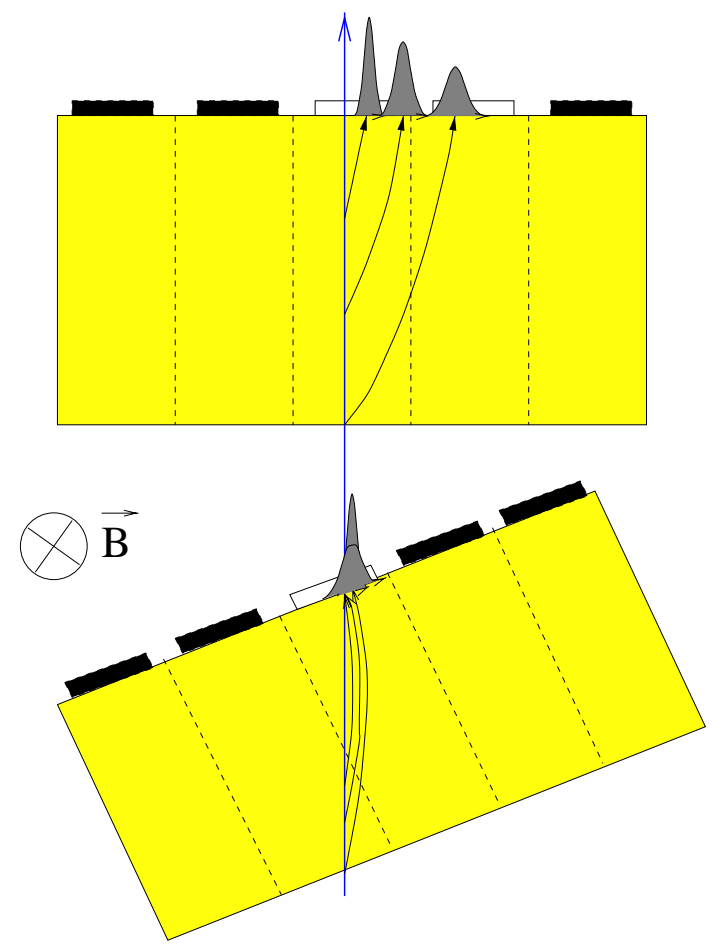

Figure 6: Drift of charge carriers under the influence of a magnetic field. The upper figure shows a perpendicular particle, in the lower the incident particle crosses the sensor at the Lorentz angle.

the angular orientation of the detectors.

The Lorentz angle for irradiated and unirradiated sensors was determined by measuring the minimum of the mean cluster size plotted as a function of the angle of the incident beam particles. For each angle, two measurements were performed in sequence. Data were taken with magnetic field off and with magnetic field on. The values of magnetic field are listed in Table 2. The mean cluster size was computed by applying minimal cuts in order to avoid biases. The dominant uncertainty on the mean cluster size for each angle is systematic. It was conservatively taken as half of the maximum difference between pairs of mean cluster sizes measured at opposite angles for data taken without the magnetic field. In fact, with no magnetic field any difference in mean cluster sizes at opposite angles is attributable to alignment uncertainties.

The mean cluster size as a function of the angle for an unirradiated sensor operated at $150 \mathrm{~V}$ in a magnetic field $B=1.48 \mathrm{~T}$ is shown in Fig. 7. The minimum is extracted by fitting the mean cluster size distribution with a parabola. Data taken without magnetic field is used to check systematic errors caused by the angular positioning of the sensor. In this case the minimum was expected at $0^{\circ}$ and any deviation from this value was used to

5) The drift trajectories are not straight lines because the electric field is not constant inside the depleted region of the sensor; see section 5.1 
correct the measurement; the value of the correction was finally quoted as a systematic error. The Lorentz angle was measured to be $9.0 \pm 0.4 \pm 0.5^{\circ}$, where the former error comes from the fit (containing the statistical and systematic error on mean cluster size described above) and the latter from the subtraction of the value found for data without magnetic field. In Fig. 8 the data for a sensor irradiated to a fluence of $10^{15} \mathrm{n}_{\mathrm{eq}} / \mathrm{cm}^{2}$ and operated at $600 \mathrm{~V}$ in $B=1.01 \mathrm{~T}$ are shown. The corresponding Lorentz angle is $3.1 \pm 0.4 \pm 0.6^{\circ}$. A similar measurement using a sensor irradiated to a fluence of $0.5 \times 10^{15} \mathrm{n}_{\mathrm{eq}} / \mathrm{cm}^{2}$ operated in a magnetic field $B=0.95 \mathrm{~T}$ yielded $2.6 \pm 0.2 \pm 0.3^{\circ}$ when it was biased at $600 \mathrm{~V}$ and $5.9 \pm 1.0 \pm 0.3^{\circ}$ when biased at $150 \mathrm{~V}$ (Table 2 ).

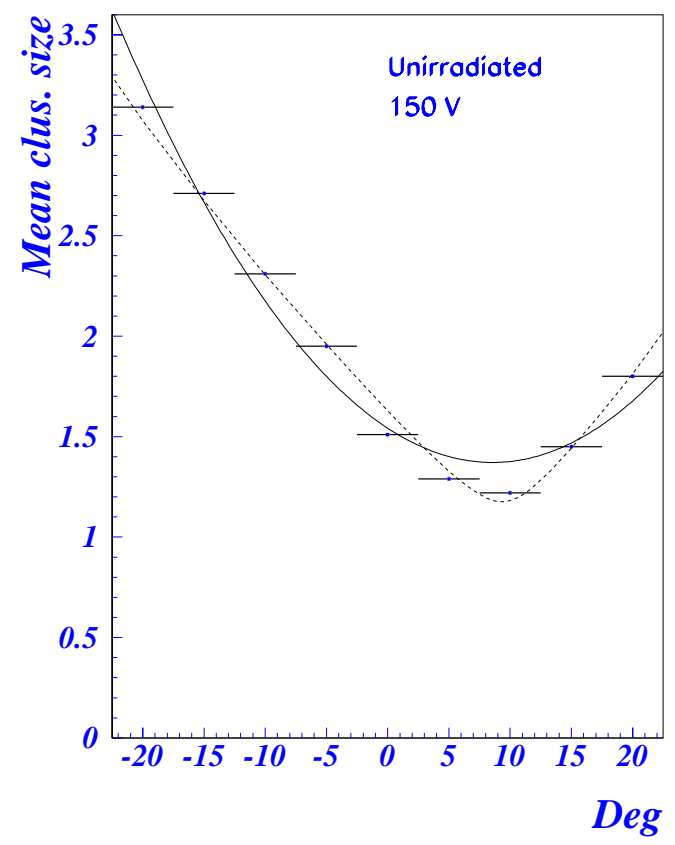

Figure 7: Mean cluster size as a function of the track angle of incidence in a magnetic field of $1.48 \mathrm{~T}$ for an unirradiated sensor. The solid line corresponds to the parabola fit. Model prediction is superimposed (dashed line).

\subsection{Interpretation of the measurements of Lorentz angle}

The measured values of Lorentz angle were compared (Fig. 9) with the predictions of a model [1] that was developed to evaluate the charge drift in silicon and to compute the mean cluster multiplicity as a function of the incidence angle using the properties of the detectors under study (temperature, magnetic field, bias voltage, depletion depth, geometry, thresholds). The Lorentz angle $\Theta_{L}$ is given by [9] $\operatorname{tg} \Theta_{L}=\mu_{H} B=r \mu_{d} B$ where $\mu_{H}$ is the Hall mobility and $\mu_{d}$ the drift mobility. Their ratio $r$ (Hall factor) is a pure number of value $\approx 1$. This depends on the scattering cross section of the charge-carriers. More specifically [10] $r=<\tau^{2}>/<\tau>^{2}$ where $\tau$ is the mean free time between collisions. It can be computed using the dependence of $\tau$ on charge-carriers energy and their energy distribution.

The Hall factor of electrons in silicon has been measured and computed at different temperatures and doping levels $[11,12]$. It has a weak dependence on temperature and does 


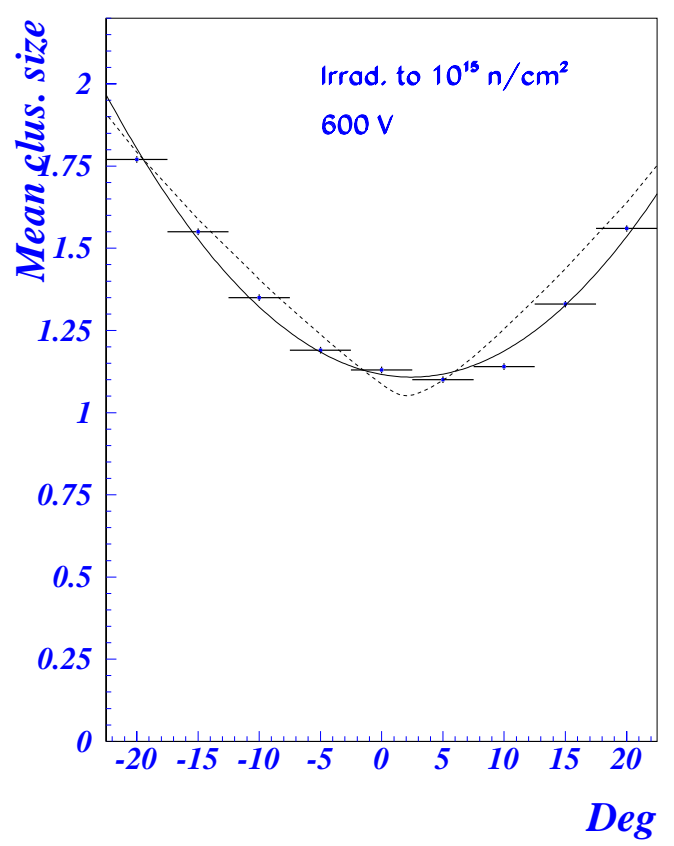

Figure 8: Mean cluster size as a function of the track angle of incidence in a magnetic field of $1.01 \mathrm{~T}$ for a sensor irradiated to a fluence of $10^{15} \mathrm{n}_{\text {eq }} / \mathrm{cm}^{2}$. The solid line corresponds to the parabola fit. Model prediction is superimposed (dashed line).

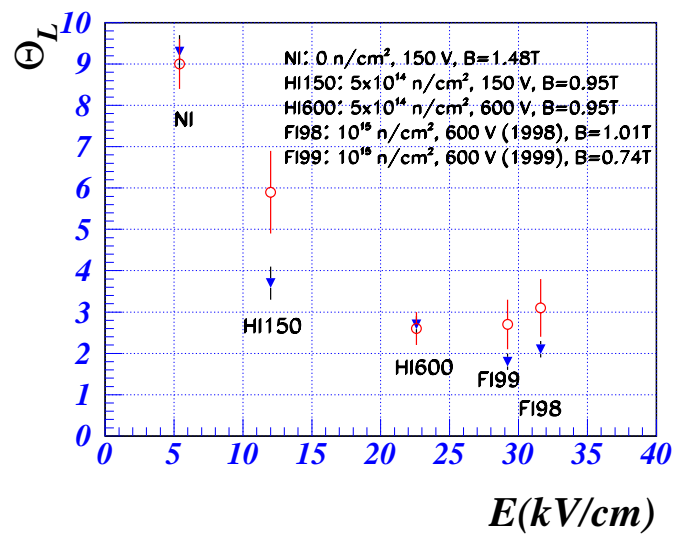

Figure 9: Lorentz angle measurement results. The dots are the experimental results, triangles are the expected values given by the model as a function of the mean electric field.

not depend on doping concentration for values below $10^{14} \mathrm{~cm}^{-3}$. The detectors tested, including those irradiated to $10^{15} \mathrm{n}_{\text {eq }} / \mathrm{cm}^{2}$, are expected to have an impurity concentration too low to affect the value of $r$. The values used are $r=1.15$ at $300{ }^{\circ} \mathrm{K}$ and $r=1.12$ at $264{ }^{0} \mathrm{~K}$. The mobility depends on temperature and electric field which vary considerably in the operation of irradiated sensors (Fig. 10), while it does not depend on impurity concentration $[13,14]$ for values below $10^{14} \mathrm{~cm}^{-3}$. This dependence is parametrized as [15] 


$$
\begin{gathered}
\mu_{d}=\frac{v_{s} / E_{c}}{\left[1+\left(E / E_{c}\right)^{\beta}\right]^{1 / \beta}} \\
\left\{\begin{array}{l}
v_{s}=1.53 \cdot 10^{9} \cdot T^{-0.87} \mathrm{~cm} \mathrm{~s}^{-1} \\
E_{c}=1.01 \cdot T^{1.55} \mathrm{~V} \mathrm{~cm} \\
\beta=2.57 \cdot 10^{-2} \cdot T^{0.66}
\end{array}\right.
\end{gathered}
$$

where $\mathrm{T}$ is the absolute temperature. The uncertainty on the mobility is $5 \%$. The electric field in the depleted region of the sensor is not constant due to the presence of the space charge. Furthermore the space charge density varies during the detector irradiation, changing its sign after type inversion. This implies that the Lorentz angle changes from point to point inside the detector and as a consequence the charge carriers drift along curved paths (Fig. 6). The effective doping concentration is assumed to be uniform. This is in agreement with the scaling of depletion depth with bias voltage observed in the detectors irradiated to $0.5 \times 10^{15} \mathrm{n}_{\mathrm{eq}} / \mathrm{cm}^{2}$. The depletion depth approximately doubles when the voltage is increased from $150 \mathrm{~V}$ to $600 \mathrm{~V}$, as expected for uniform doping. With this assumption for irradiated sensors, which have a p-type conductivity and are not completely depleted, the electric field varies linearly from $2 \cdot V / d$ near the pixel (where the $n-p$ junction is located) to zero at the limit of depleted zone. In unirradiated devices which are over-depleted, the electric field varies linearly from $\left(V-V_{d}\right) / d$ near the pixels to $\left(V+V_{d}\right) / d$ at the junction located on the backside (where $V=150 \mathrm{~V}$ was the applied bias). The full depletion voltage was determined to be $105 \mathrm{~V}$ for an unirradiated sensor. The effective Lorentz angle was defined as the incident track angle for which the mean hit multiplicity is at its minimum. In the model, to calculate the cluster multiplicity, the ionization charge was transported to the pixel plane taking into account the electric and magnetic forces and diffusion. The transverse diffusion coefficient for an electric field parallel to the $<111>$ direction is given in [15]. Since it has only a weak dependence on the electric field $E$ a value of $25 \mathrm{~cm}^{2} \mathrm{~s}^{-1}$ (independently of $E$ ) was taken. Finally, to define hit pixels, a threshold is applied on the charge collected by every pixel. The values of depletion depth used in the model were experimentally measured (see Section 4), so that all except one of the model parameters are measured quantities. The only fit parameter was the threshold ${ }^{6)}$. This was determined using data with no magnetic field present. The uncertainty on the values given by the model was estimated by varying the input depletion depths, the magnetic field and the mobility within their errors. Other potential sources of errors proved to be negligible. Fig. 10 shows the mobility as a function of the electric field and for $T=264{ }^{\circ} \mathrm{K}$ and $T=300{ }^{0} \mathrm{~K}$, which were the operating temperatures of irradiated and unirradiated detectors. The markers correspond to the temperature and the mean electric field $\langle E\rangle=V / d$ present in the detectors under study.

The experimental results presented in the previous section were compared with the prediction of the model in Fig. 9 and summarized in Table 2. Taking into account the variation of the magnetic field, it is apparent that for irradiated sensors the Lorentz angle is smaller mostly because of the larger electric field. The dependence of Lorentz angle on electric

6) It was necessary to fit the threshold instead of using the value obtained by electronic calibration of the front end electronics because the sensors used for the measurements had a non uniform charge collection with losses along the edges of the pixel cells ([16]). This necessitated the extraction of an effective threshold from the data (which was about $20 \%$ higher than the real one). 


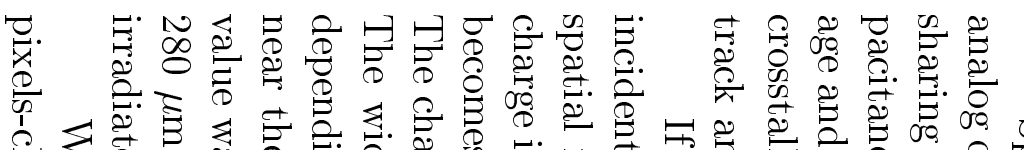

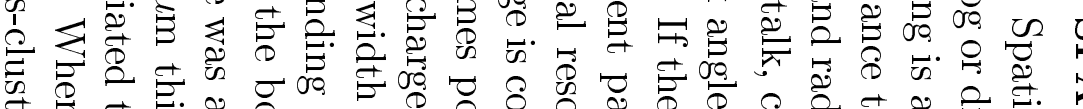

焉

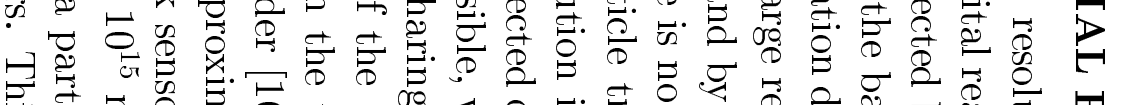

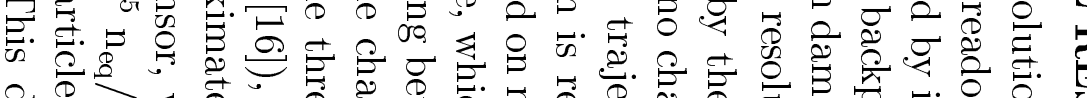

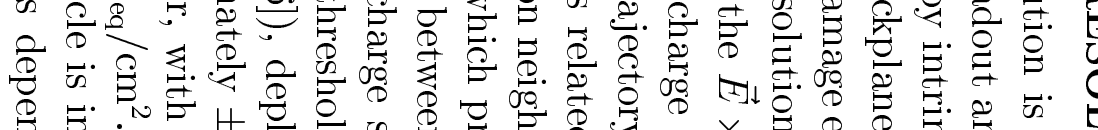

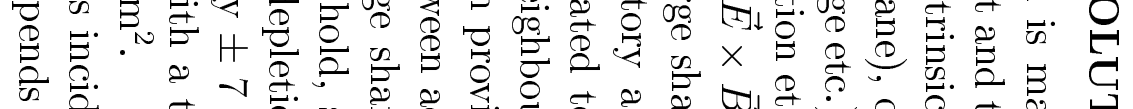

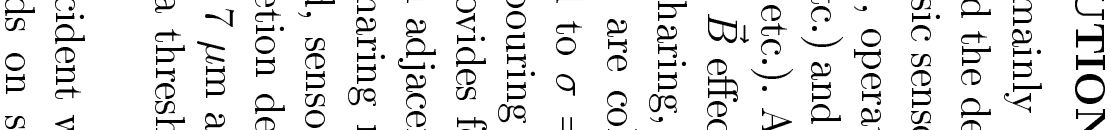

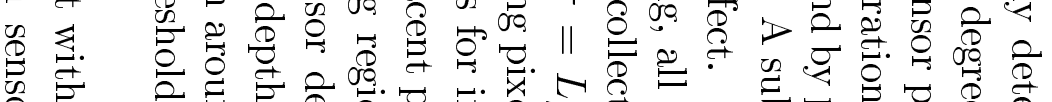

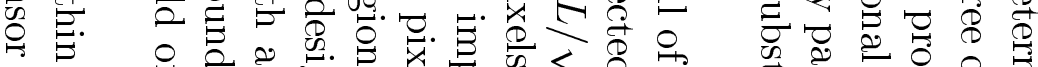

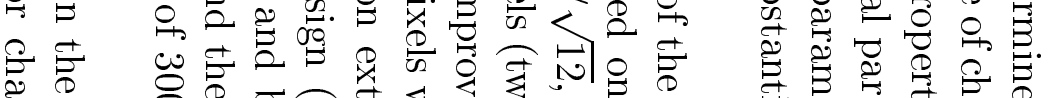

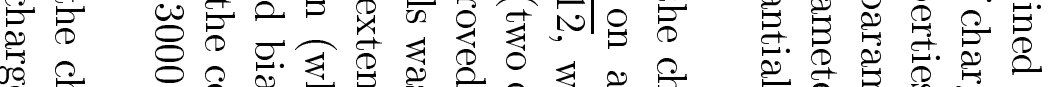

o.

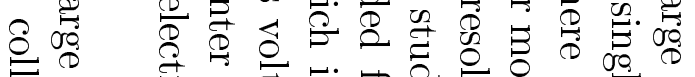

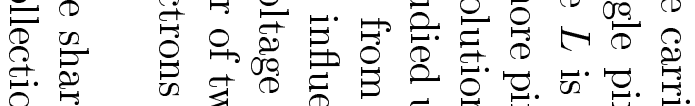

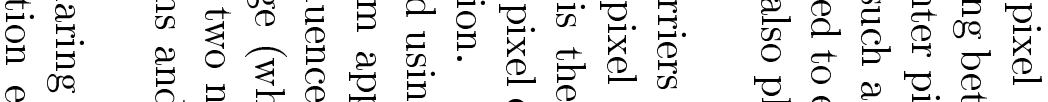

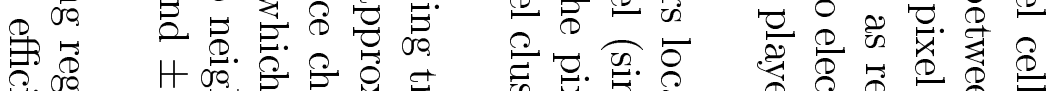

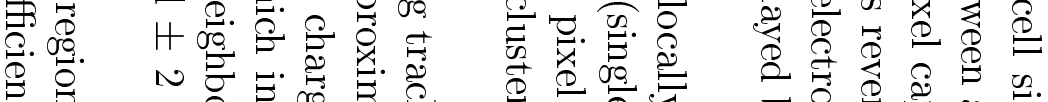

蛋

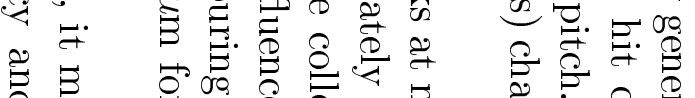

穴苔.

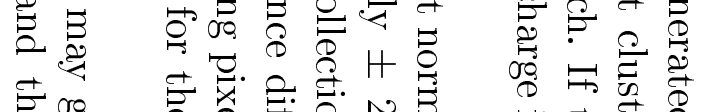

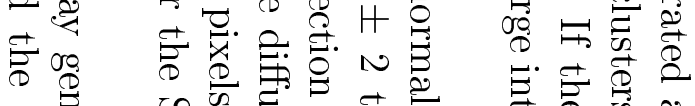

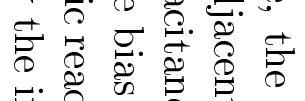

ㄱ.ㄱ.

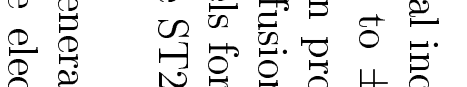

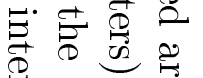

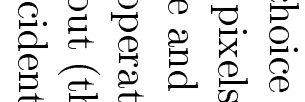

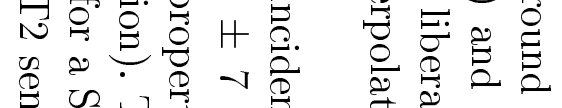

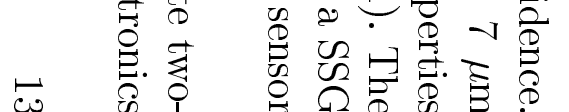

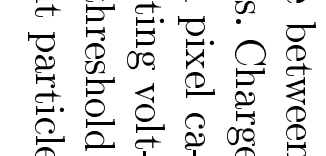

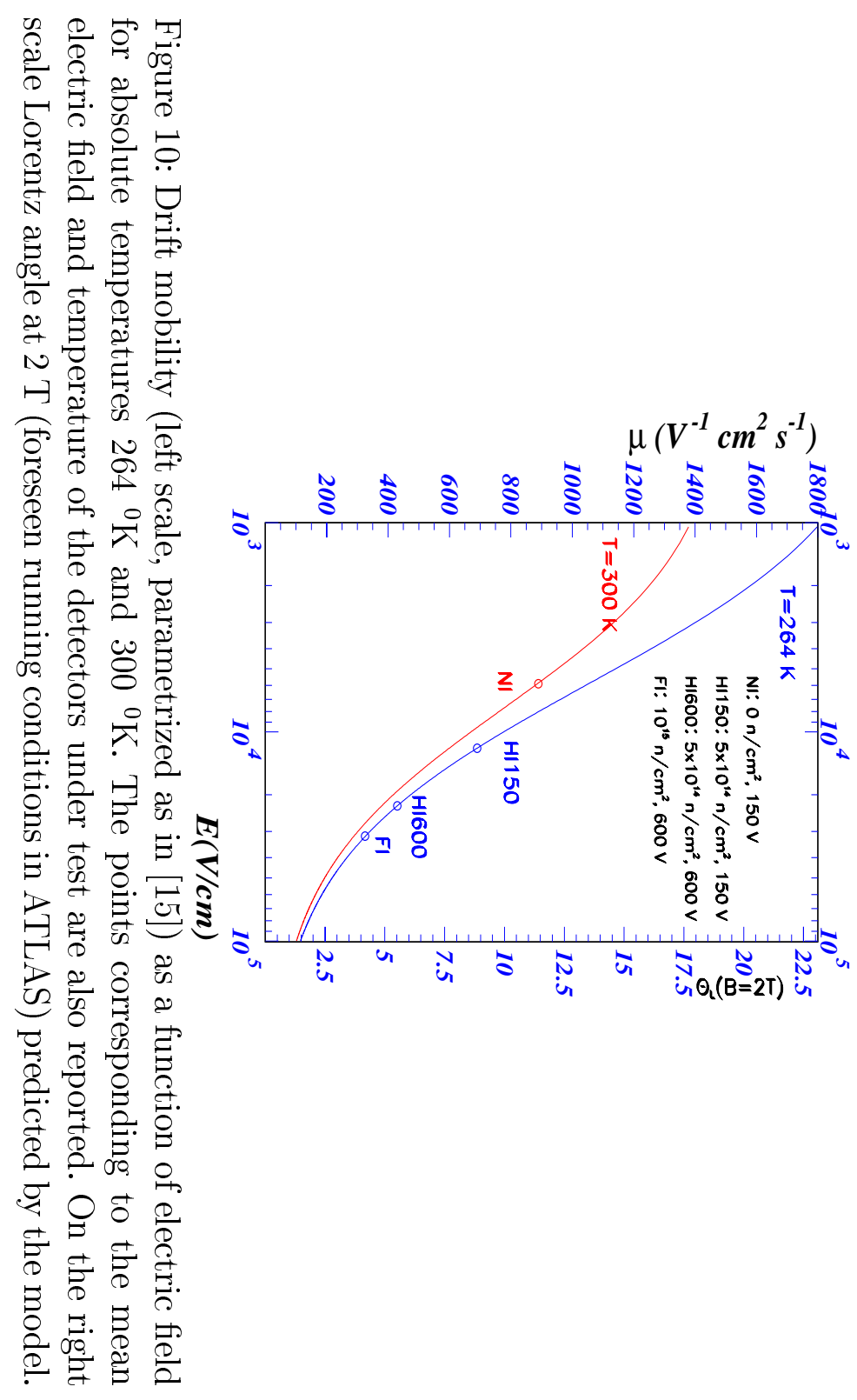

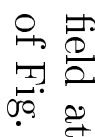

官宫

$\exists$

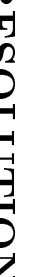


threshold. Two different algorithms were used to reconstruct the spatial position of twopixels-clusters. A digital algorithm which uses the center position between the two pixels and an analog algorithm that corrects the binary position just described using an interpolation of the charge collected by the two pixels. Since it was observed that the ratio of the charge collected on the right hand side pixel $\left(Q_{r}\right)$ over the total charge collected by the two pixels $\eta=Q_{r} /\left(Q_{l}+Q_{r}\right)$ (where $Q_{r}$ and $Q_{l}$ are the charges collected by the right hand side and left hand side pixels in the cluster respectively) had a dependence on the position of the passing particle, the following interpolation was adopted [17]:

$$
x_{\mathrm{an}}=x_{\operatorname{dig}}+\frac{\Delta}{N_{0}} \int_{0}^{\eta} \frac{d N}{d \eta} d \eta
$$

where $x_{\text {an }}$ and $x_{\text {dig }}$ are the spatial positions reconstructed by the analog and digital algorithms respectively. This formula assumes that $N_{0}$ particles are spread uniformly over an interval $\Delta$ that is the width of the region within which charge sharing occurs.

An equivalent procedure was adopted for multi-pixel-clusters. These occur when particles traverse the pixel sensor at an angle. For inclined particles, the charge is collected over a region approximately given by $\mathrm{D} \times \operatorname{tg}(\phi)$, where $\mathrm{D}$ is the sensor depletion depth. Charged particles with large incident angles produce signals on many pixels and the average charge per pixel decreases, despite the longer trajectory in the silicon. Since only the signal amplitudes on the edge pixels in the clusters carry information on the position of the passing particle, the digital and the analog algorithms described above were used to reconstruct the coordinate but only taking into account the first and the last pixel in the clusters [18].

Referring to (2), $\Delta$ depends on angle, cluster multiplicity and sensor design and is extracted from a fit to data for each configuration.

Fig. 11 shows the correlation between $\eta$ and the difference between the reconstructed track position and the space point recostructed with digital information alone at an angle of $15^{\circ}$ for an ST1 sensor. The figure also shows that the correction to be applied depends on the number of pixels in the cluster (i.e. the cluster multiplicity), so it is convenient to treat different multiplicities separately.

As the track length under a pixel is geometrically limited by $p / \sin \alpha$ ( $p$ being the pixel size), charges on a pixel exceeding $Q_{\text {cut }}=\lambda p / \sin \alpha$ (where $\lambda$ is the mean number of electrons generated per unit path length) are due to energy loss fluctuations and $\delta$ electrons. The impact of these fluctuations on resolution was reduced by setting pulse heights exceeding $Q_{\text {cut }}$ to $Q_{\text {cut }}$, when computing $\eta$.

In what follows, $\mathrm{x}$ describes the short $(50 \mu \mathrm{m})$ and $\mathrm{y}$ the long $(400 \mu \mathrm{m})$ pitch dimension of the pixel assembly.

\subsection{Telescope resolution}

Spatial resolution was determined by computing the residuals between the coordinate measured by the pixel detector and that predicted by the silicon microstrip telescope. The extrapolation uncertainty depends on many parameters, e.g. the position of the microstrip planes and of the pixel detector under study, the microstrips intrinsic resolution, the amount of material along the beam path etc. Telescope resolution was improved applying a tighter selection on track reconstruction $\chi^{2}$ probability. Keeping $20 \%$ of events, telescope resolution improved from $\sim 6$ to $\sim 4 \mu \mathrm{m}$. In order to study the telescope resolution, data taken at normal incidence were used. At normal incidence mainly single-pixel 


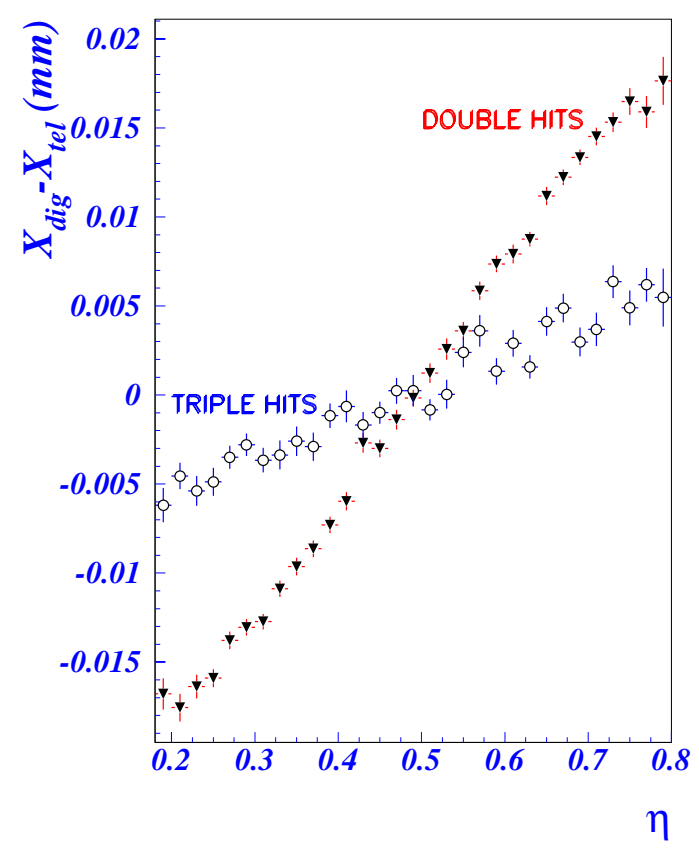

Figure 11: Difference between position measured by the telescope and by the pixel detector with digital information alone, plotted against the variable $\eta$ (defined in the text). Data are taken with an ST1 device for an angle of incidence of $15^{\circ}$.

and double-pixel-clusters occur. The resolution is determined by their relative abundance. Single pixel and double pixel clusters were studied separately. Examples of such residual distributions are shown for a modified SSGb $200 \mu \mathrm{m}$ thick sensor in Fig. 12. Single-pixel clusters occur when incident particles cross the pixel central region of with $\mathrm{L}=\mathrm{p}-2^{*} \Delta$. Then for single pixel clusters the distribution is parametrized with a uniform distribution of width L, convoluted with a Gaussian distribution that takes into account the resolution of the silicon strip telescope, threshold effects and $\delta$-rays. The result of this fit (Fig. 12c) was $\sigma_{\text {tel }}=4.5 \pm 0.3 \mu \mathrm{m}$ and $\mathrm{L}=44.0 \pm 0.4 \mu \mathrm{m}$ for the extension of the region at the centre of the pixel where the charge is collected by a single pixel. The fitted width $\mathrm{L}$ is in agreement with the measured width of $\pm 3 \mu \mathrm{m}$ for the region in which charge sharing occurs for this sensor design and operating conditions. As a consequence of the dimensions of the region of charge sharing, the double-pixel-cluster distribution of residuals had a width of $5.2 \mu \mathrm{m}$ for the digital algorithm (Fig. 12a), when calculated fitting the distributions with a Gaussian function. An alternative method to estimate telescope resolution is to fit a Gaussian function to the analog residuals. This yielded a value of $4.8 \mu \mathrm{m}$ (Fig. 12b), in agreement within statistical errors with the value obtained using the fit to the single pixel cluster distribution.

At $0^{\circ}$ telescope resolution values between 3 and $5 \mu \mathrm{m}$ were measured, depending on the different amount of material along the beam line. At higher angles slightly worse values were measured, due to the projection on the pixel detector plane (which yields a telescope resolution proportional to $1 / \cos \alpha$ ) and the presence of more material along the beam when detectors are tilted.

The resolution figures quoted here are not corrected for telescope extrapolation un- 

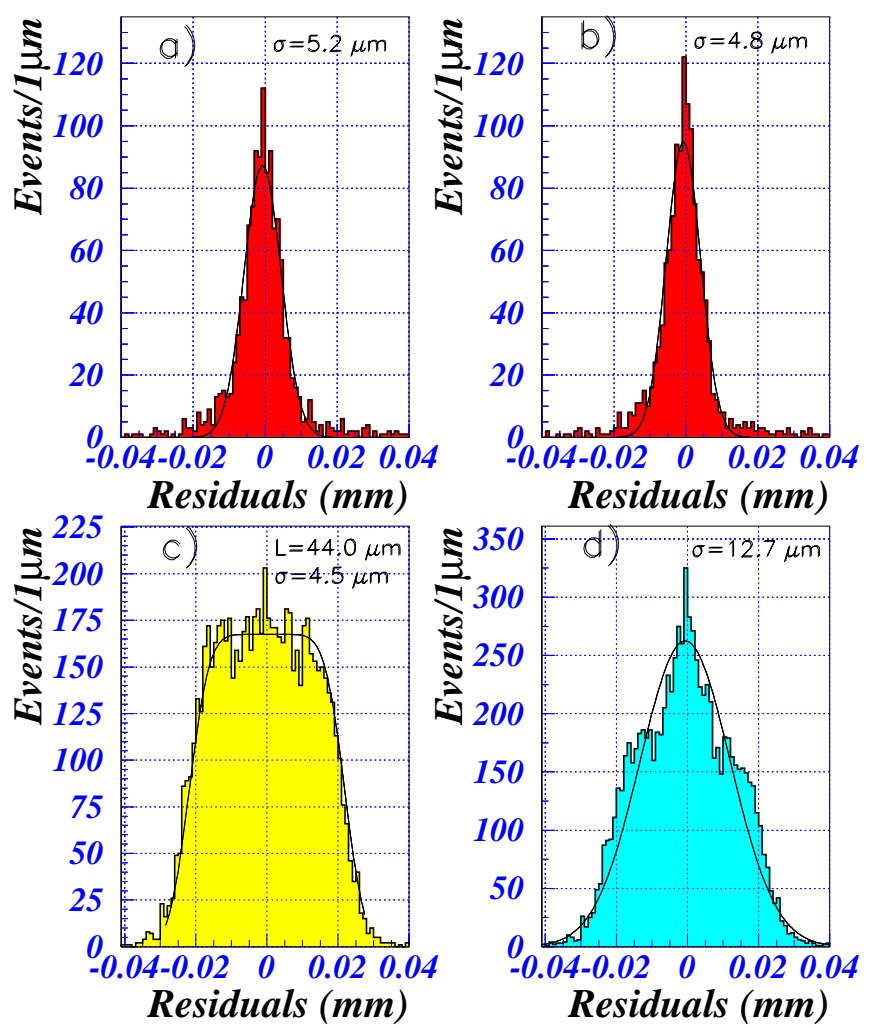

Figure 12: Residuals between position measured by the telescope and by pixel detector for a $200 \mu \mathrm{m}$ thick SSGb sensor at $0^{\circ}$. a) Double hits residuals (digital algorithm). b) Double hits residuals (analog algorithm). c) Single hits residuals. d) Total residuals distribution (analog algorithm).

certainty since its measurement may be affected by systematic effects which are difficult to evaluate for any data taking configuration. The quoted values are the standard deviations evaluated by fitting the residual distributions with a Gaussian function. These are less sensitive to statistical fluctuations than the rms and give a reasonably good description of the width of the distributions even when the distributions are not Gaussian (as occurs at angles at which a limited charge sharing is present).

\section{2 $\mathrm{x}$-spatial resolution at normal incidence}

At $0^{\circ}$ the resolution depends on the relative fraction of single and multi-hit clusters and is dominated by the single hit cluster resolution. The combined distribution of single and double pixel clusters for the SSGb $200 \mu \mathrm{m}$ thick sensor (Fig. 12d) has a standard deviation of $12.7 \mu \mathrm{m}$ (the $\mathrm{rms}$ is $14.0 \mu \mathrm{m}$ ).

The relative weight of single-pixel and double-pixel-clusters are listed in Table 3, where the results for other six sensors with different designs or with different irradiation are presented. There is not a great difference recorded between analog and digital resolutions for a given sensor, but differences appear between different configurations. Devices which collected more charge (either due to their larger thickness, or because they were fully depleted or since the design had negligible charge loss, as the ST1 and SSG layouts) 
produced less single pixel clusters, located in a narrower region (i.e. L was smaller) and therefore had better resolution. As a consequence unirradiated $280 \mu \mathrm{m}$ thick devices provided resolutions superior to the irradiated sensors or to the $200 \mu \mathrm{m}$ thick sensors. The ST2 devices had worse resolution than SSG and ST1 sensors, as a consequence of charge collection inefficiencies.

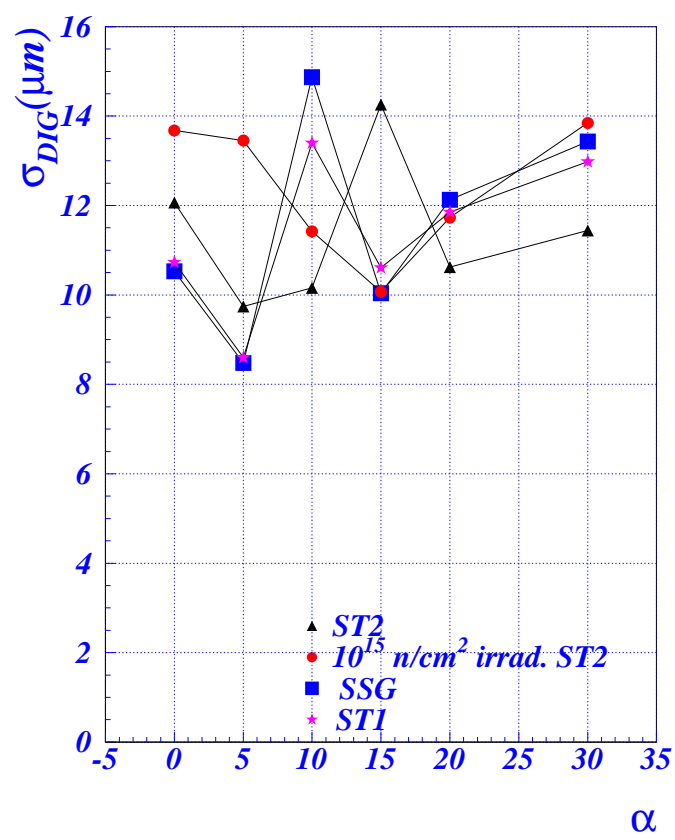

Figure 13: Measured digital resolution without subtraction of telescope extrapolation uncertainty.

\section{3 x-spatial resolution as a function of the angle of incidence}

Finally, the dependence of the resolution on the angle $\alpha$ of the incident particle with respect to the normal to sensor surface was studied. The standard deviations of the all-cluster residual distributions are shown in Fig. 13 (using a digital algorithm) and in 14 and 15 (analog algorithm). The data were not corrected for the silicon microstrip telescope extrapolation uncertainty.

As the tilt angle is increased, the fraction of double pixel clusters increases, their residual distribution gets wider and the single pixel cluster distribution narrower. This is a consequence of single pixel clusters occurring in a more restricted region.

The best digital resolution is obtained when the two distributions are equally populated. At any given angle about $98 \%$ of clusters are formed from only two multiplicities (1 and 2, 2 and 3 and so on, depending on the angle). When they are equally populated the digital resolution is of the order of $p / 2 / \sqrt{12}=25 \mu \mathrm{m} / \sqrt{12}$. When the angle is such that nearly all of the events belong to one multiplicity only, the digital resolution is of the order of $p / \sqrt{12}=50 \mu \mathrm{m} / \sqrt{12}$. Then the digital resolution as a function of angle (Fig. 13) shows a structure of alternating minima and maxima. The angular position of minima and maxima depends on the charge collection properties of the device which influence the cluster multiplicity. For example, for $280 \mu \mathrm{m}$ thick sensor devices and with negligible charge loss (SSG and ST1), the position of first minimum is obtained at $5^{\circ}$. 


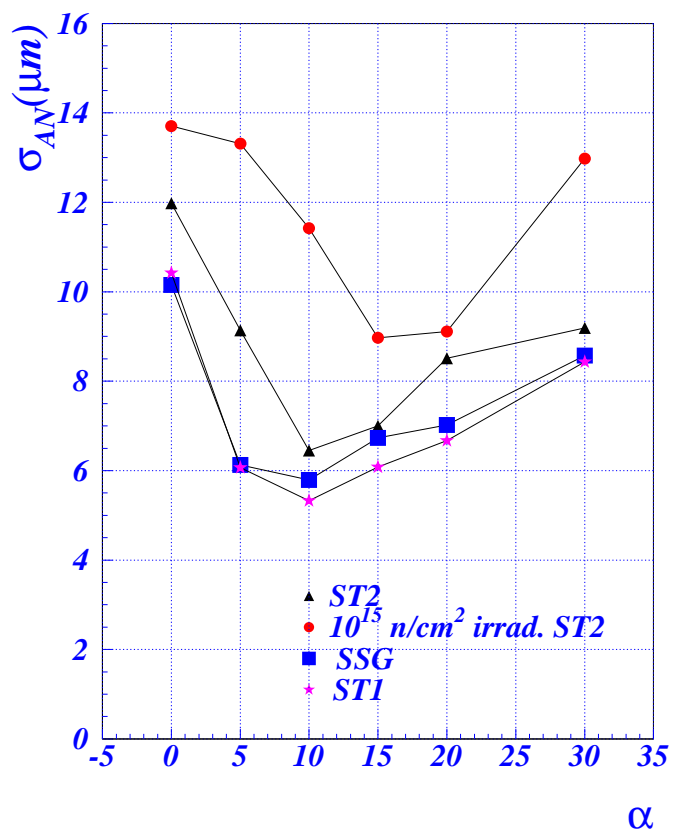

Figure 14: Measured analog resolution without subtraction of telescope extrapolation uncertainty (1998 data).

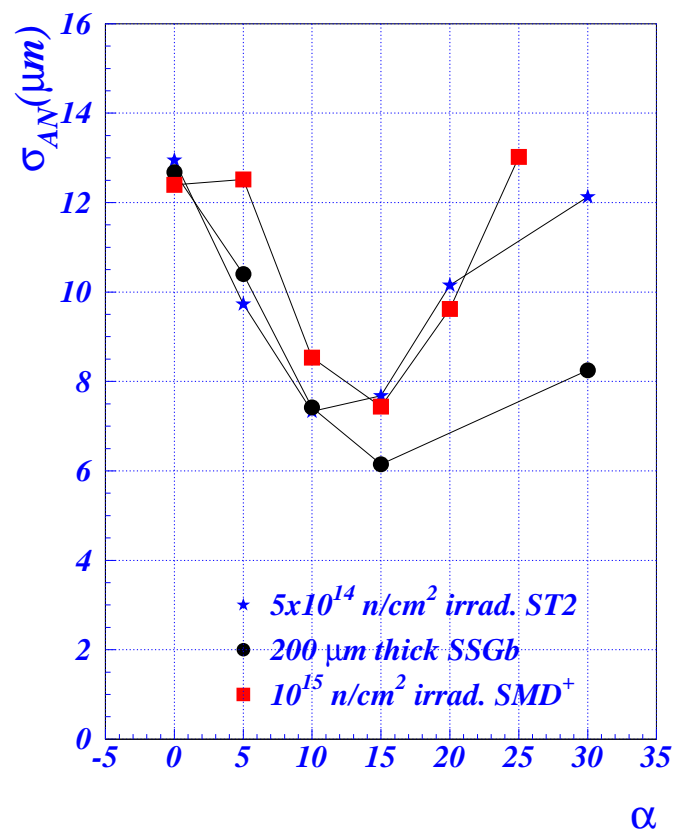

Figure 15: Measured analog resolution without subtraction of telescope extrapolation uncertainty (1999 and 2000 data).

This angle is larger for the ST2 design, since it suffers from charge loss and larger still for an irradiated ST2 sensor operating underdepleted. The resolutions at the maxima and minima are governed by pixel pitch only. 
The spatial resolution obtained with the analog algorithm (Fig. 14 and 15) is always better than the corresponding digital resolution once the incidence angles are larger than $0^{\circ}$. The best resolution value occurs when the proportion of single pixel clusters becomes negligible. This occurred at $10^{\circ}$ for the unirradiated $280 \mu \mathrm{m}$ thick devices $(5.3 \mu \mathrm{m}$ before correction for the telescope resolution, $3.2_{-1.2}^{+0.8} \mu$ m after correction ${ }^{7)}$ ), and at $15^{\circ} \div 20^{\circ}$ for partially depleted or thinner sensors. The best resolution before subtraction of telescope error for a device irradiated to $10^{15} \mathrm{n}_{\mathrm{eq}} \mathrm{cm}^{-2}$ is $7.4 \mu \mathrm{m}, 6.0_{-0.6}^{+0.5} \mu \mathrm{m}$ after subtraction ${ }^{7)}$. It should be noted that the spatial resolution is not significantly degraded post-irradiation, showing that there are no inhomogenities in the sensor after irradiation. The differences in spatial resolution before and after irradiation are completely explained in terms of reduced charge collection efficiency.

As the angle of incidence increases further, the charge collected by every pixel is reduced and energy loss fluctuations introduce inefficiencies in the first and last pixel in the cluster, thus degrading the resolution.

Similar results were obtained for all the designs. For any given design, no differences were found between differing implant widths, for a given degree of charge collection.

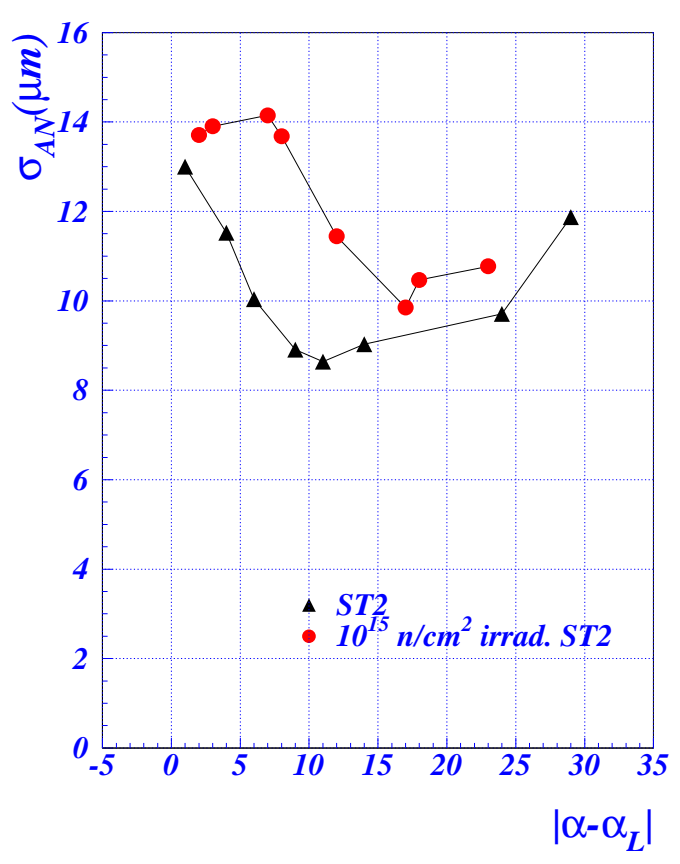

Figure 16: Measured analog resolution inside a magnetic field as a function of the difference between the beam incidence angle and the Lorentz angle, without subtraction of telescope extrapolation uncertainty.

\subsection{Spatial resolution in the presence of a magnetic field}

It is expected that the effect of a magnetic field on the resolution is to shift the angular dependence by a quantity equal to the Lorentz angle. In Fig. 16 the analog resolution for data taken with a magnetic field is shown as a function of the absolute value of the difference between the incidence angle and the measured Lorentz angle. The

\footnotetext{
7) Statistical error.
} 


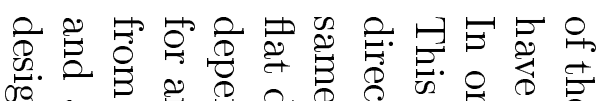

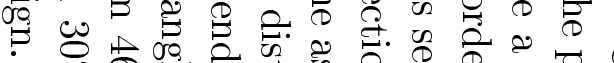

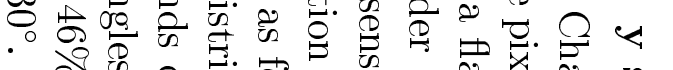

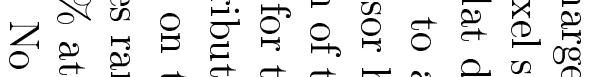

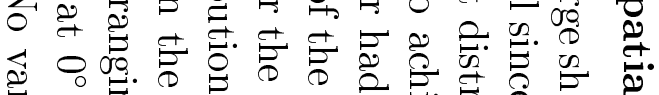
.

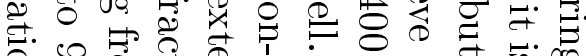

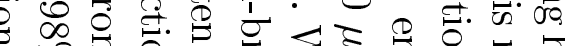

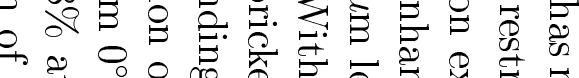

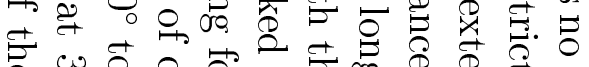

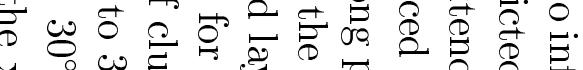

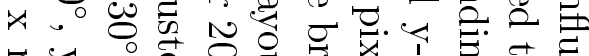

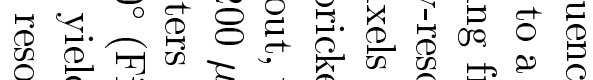
船 E.

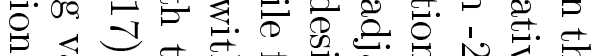

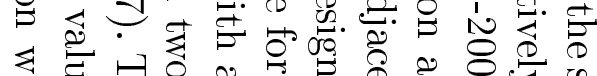

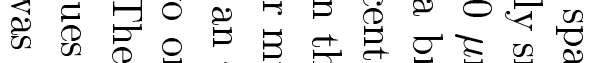

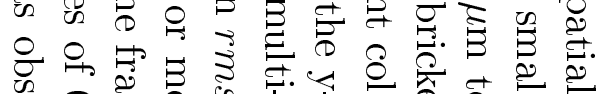

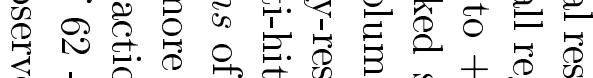

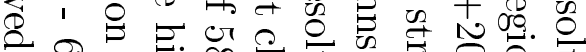

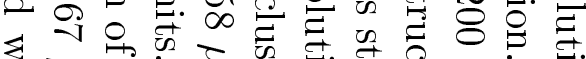

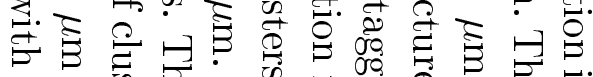

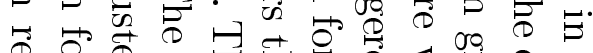

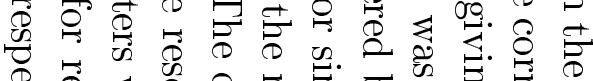

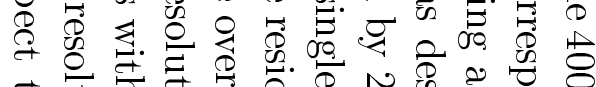

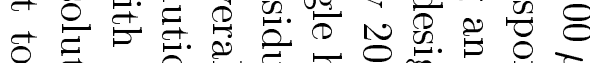

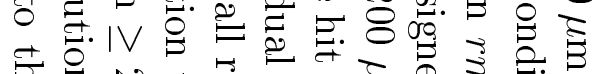

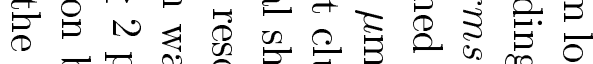

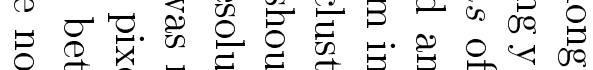

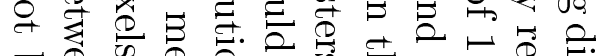
马.

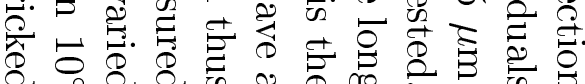

ن

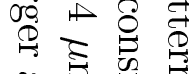

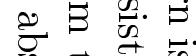
क $0 \stackrel{0}{\circ}$

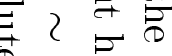
○ 0 हैं ণ 룰

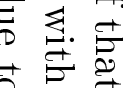
๑ 뭉 군. Q 용 Е. 过 융 疍 $\Xi$. 施官 दे $\overrightarrow{0}$ 苟. 疍 유융

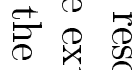

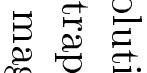
哭 웅

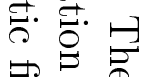
बै 눙

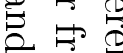
ㅇํㄹ 
electric field to increase further to values in excess of $10^{4} \mathrm{~V} / \mathrm{cm}$. Since in this regime the electron drift mobility is a rapidly decreasing function of the electric field, the Lorentz angle is consequentely affected. No deviations from expectations have been found at 264 ${ }^{0} \mathrm{~K}$ and $300{ }^{0} \mathrm{~K}$.

A Lorentz angle variation is then expected during operation in ATLAS, when the bias will be adjusted for the increased depletion voltage. This will affect the ultimate spatial resolution, due to its dependence on track incidence angle. However a position resolution optimal value better than $7.4 \mu \mathrm{m}$ at $15^{\circ}$ has been demonstrated even after irradiation of $1 \times 10^{15} 1 \mathrm{MeV} \mathrm{n}$ eq $/ \mathrm{cm}^{2}$, when measured charge is properly taken into account.

\section{References}

[1] M. Aleppo, T. Lari Contrib. to the Intern. Workshop on Semiconductor Pixel Detectors for Particles and X-rays, PIXEL 2000, Genoa, June 2000, to be published on Nucl. Instr. and Meth. A

[2] The ATLAS Pixel Collaboration, ATLAS Pixel Detector Technical Design Report, LHCC 98/13, CERN 1998

[3] M.S.Alam et al., Nucl. Instr. and Meth. A 456 (2001) 217

T. Rohe et al., Nucl. Instr. and Meth. A 409 (1998) 224.

The ATLAS Pixel Collaboration, Electrical Characteristics of Silicon Pixel Sensors, to be published on Nucl. Instr. and Methods

[4] G. Batignani et al., Nucl. Instr. and Meth. A 277 (1989) 147

[5] R.H. Richter et al., Nucl. Instr. and Meth. A 377 (1996) 412

[6] R. Wunstorf, Contrib. to the 4th Int. Symp. on Development and Application of Semiconductor Tracking Detectors, Hiroshima, March 2000, to be published on Nucl. Instr. and Meth., section A

[7] L. Blanquart et al., Nucl. Instr. and Meth. A 439 (2000) 403

[8] K. Einsweiler et al., IEEE Trans. on Nuclear Science 46 (1999) 166 and 792

[9] W. Shockley, Electrons and holes in semiconductors, Van Nostrand, Princeton, N.J. (1950)

[10] S. M. Sze, Physics of semiconductor devices, 2nd Edition, Wiley-interscience, New York (1981)

[11] I. G. Kirnas et al., Phys. Stat. Sol. A 23 (1974) K123

[12] P. Blood and J. Worton, Rep. Prog. Phys. 41 (1978) 157

[13] V. Eremin and Z. Li, NIM A 362 (1995) 338

[14] T. J. Brodbeck et al., Carrier mobilities in irradiated silicon, Contrib. to the 5th Conference on Position Sensitive Detectors, London, Sep. 1999, to be published on NIM, section A

[15] C. Jacoboni et al., Solid State Electronics 20 (1977) 77

[16] F. Ragusa, Nucl. Instr. and Meth. A 447 (2000) 184

[17] E. Belau et al., Nucl. Instr. and Meth. A 214 (1983) 214

[18] R. Turchetta, Nucl. Instr. Meth. A 335 (1993) 44

C. Troncon, IEEE Trans. on Nucl. Science, vol 47 (2000), 125 
Table 1: Depletion depth measurement results.

\begin{tabular}{|c|c|c|c|}
\hline Fluence $\left[\mathrm{n}_{\mathrm{eq}} / \mathrm{cm}^{2}\right]$ & Thickness $[\mu \mathrm{m}]$ & Bias Voltage [V] & Depletion depth $[\mu \mathrm{m}]$ \\
\hline 0 & 200 & 150 & $190 \pm 7$ \\
\hline 0 & 280 & 150 & $288 \pm 6$ \\
\hline $0.5 \times 10^{15}$ & 280 & 600 & $261 \pm 8$ \\
$0.5 \times 10^{15}$ & 280 & 400 & $252 \pm 10$ \\
$0.5 \times 10^{15}$ & 280 & 200 & $147 \pm 7$ \\
$0.5 \times 10^{15}$ & 280 & 150 & $123 \pm 11$ \\
$0.5 \times 10^{15}$ & 280 & 100 & $100 \pm 7$ \\
\hline $10^{15}(1998)$ & 280 & 600 & $189 \pm 12$ \\
$10^{15}(1999)$ & 280 & 600 & $217 \pm 13$ \\
$10^{15}$ & 280 & 300 & $111 \pm 10$ \\
\hline
\end{tabular}


Table 2: Lorentz angle measurement results.

\begin{tabular}{|l|c|c|c|c|c|}
\hline Fluence $\left[\mathrm{n}_{\text {eq }} / \mathrm{cm}^{2}\right]$ & 0 & $0.5 \times 10^{15}$ & $0.5 \times 10^{15}$ & $1 \times 10^{15}(1998)$ & $1 \times 10^{15}(1999)$ \\
Bias Voltage $[\mathrm{V}]$ & 150 & 150 & 600 & 600 & 600 \\
$T\left[{ }^{0} \mathrm{~K}\right]$ & 300 & 264 & 264 & 264 & 264 \\
Depletion $[\mu \mathrm{m}]$ & $288 \pm 6$ & $123 \pm 11$ & $261 \pm 8$ & $189 \pm 12$ & $217 \pm 13$ \\
Magn. Field [T] & $1.48 \pm 0.02$ & $0.95 \pm 0.05$ & $0.95 \pm 0.05$ & $1.01 \pm 0.05$ & $0.74 \pm 0.05$ \\
\hline$\Theta_{L}$ (meas.) $\left.{ }^{\circ}\right]$ & $9.0 \pm 0.4 \pm 0.5$ & $5.9 \pm 1.0 \pm 0.3$ & $2.6 \pm 0.2 \pm 0.3$ & $3.1 \pm 0.4 \pm 0.6$ & $2.7 \pm 0.4 \pm 0.4$ \\
$\Theta_{L}$ (th.) $\left[^{\circ}\right]$ & $9.3 \pm 0.4$ & $3.7 \pm 0.5$ & $2.7 \pm 0.2$ & $2.1 \pm 0.2$ & $1.8 \pm 0.2$ \\
\hline
\end{tabular}


Table 3: Measurements of Spatial Resolution* .

\begin{tabular}{|c|c|c|c|c|c|c|c|}
\hline Design & ST2 & ST1 & SSG & ST2 & ST2 & $\overline{\mathrm{SSGb}}$ & $\mathrm{SMD}^{+}$ \\
\hline Isolation & p-spray & p-stop & p-spray & p-spray & p-spray & p-spray & p-spray \\
\hline $\begin{array}{c}\text { Fluence }\left(\mathrm{n}_{\text {eq }} / \mathrm{cm}^{2}\right) \\
\operatorname{Bias}(\mathrm{V})\end{array}$ & $\begin{array}{c}0 \\
150\end{array}$ & $\begin{array}{c}0 \\
150\end{array}$ & $\begin{array}{c}0 \\
150\end{array}$ & $\begin{array}{l}10^{15} \\
600\end{array}$ & $\begin{array}{c}0.5 \cdot 10^{15} \\
600\end{array}$ & $\begin{array}{c}0 \\
150\end{array}$ & $\begin{array}{l}10^{15} \\
600\end{array}$ \\
\hline $\begin{array}{c}\text { Depletion depth }(\mu \mathrm{m}) \\
\text { Thickness }(\mu \mathrm{m})\end{array}$ & $\begin{array}{l}280 \\
280\end{array}$ & $\begin{array}{l}280 \\
280\end{array}$ & $\begin{array}{l}280 \\
280\end{array}$ & $\begin{array}{l}190 \\
280\end{array}$ & $\begin{array}{l}260 \\
280\end{array}$ & $\begin{array}{l}200 \\
200\end{array}$ & $\begin{array}{l}230 \\
250\end{array}$ \\
\hline $\begin{array}{c}\text { Cluster multiplicity (\%) } \\
\text { (at normal incidence) } \\
1 \text { hit } \\
2 \text { hits } \\
3 \text { hits } \\
4 \text { hits or more }\end{array}$ & $\begin{array}{c}76.8 \\
20.9 \\
1.2 \\
1.1\end{array}$ & $\begin{array}{c}67.5 \\
29.5 \\
1.7 \\
1.3\end{array}$ & $\begin{array}{c}66.7 \\
30.4 \\
1.6 \\
1.3\end{array}$ & $\begin{array}{c}90.6 \\
8.4 \\
0.6 \\
0.4\end{array}$ & $\begin{array}{c}83.6 \\
14.5 \\
1.3 \\
0.6\end{array}$ & $\begin{array}{c}82.5 \\
15.9 \\
0.8 \\
0.8\end{array}$ & $\begin{array}{c}85.9 \\
12.4 \\
1.0 \\
0.7\end{array}$ \\
\hline $\begin{array}{c}\text { Normal incidence: } \\
\text { Charge sharing region }(\mu \mathrm{m}) \\
\text { Digital resolution* }(\mu \mathrm{m}) \\
\text { Analog resolution* }(\mu \mathrm{m})\end{array}$ & $\begin{array}{c}9.2 \\
12.1 \\
12.0 \\
\end{array}$ & $\begin{array}{l}13.6 \\
10.7 \\
10.4 \\
\end{array}$ & $\begin{array}{l}14.0 \\
10.5 \\
10.1 \\
\end{array}$ & $\begin{array}{c}3.5 \\
13.7 \\
13.7 \\
\end{array}$ & $\begin{array}{c}5.8 \\
13.1 \\
12.9 \\
\end{array}$ & $\begin{array}{c}6.2 \\
12.7 \\
12.6\end{array}$ & $\begin{array}{c}4.3 \\
12.5 \\
12.4\end{array}$ \\
\hline $\begin{array}{l}\text { Best Analog Resolution* }(\mu \mathrm{m}) \\
\text { Corresp. angle }\end{array}$ & $\begin{array}{l}6.5 \\
10^{\circ}\end{array}$ & $\begin{array}{l}5.3 \\
10^{\circ}\end{array}$ & $\begin{array}{l}5.8 \\
10^{\circ}\end{array}$ & $\begin{array}{c}9 \\
15^{\circ}\end{array}$ & $\begin{array}{l}7.3 \\
10^{\circ}\end{array}$ & $\begin{array}{l}6.2 \\
15^{\circ}\end{array}$ & $\begin{array}{r}7.4 \\
15^{\circ}\end{array}$ \\
\hline
\end{tabular}

* Telescope extrapolation uncertainty not subtracted.

+ Oxygenated silicon substrate and moderated $p$-spray isolation. 